\title{
de Sitter black hole with a conformally coupled scalar field in four dimensions
}

\author{
Cristián Martínez, Ricardo Troncoso and Jorge Zanelli* \\ Centro de Estudios Científicos (CECS), Casilla 1469, Valdivia, Chile.
}

\begin{abstract}
A four-dimensional black hole solution of the Einstein equations with a positive cosmological constant coupled to a conformal scalar field is given. There is a curvature singularity at the origin, and the scalar field has a divergence inside the event horizon. The electrically charged solution, which has a fixed charge-to-mass ratio, is also found. The quartic self-interacting coupling constant becomes bounded in terms of Newton's constant and the cosmological constant. The solution satisfies both dominant and the strong energy conditions.
\end{abstract}

${ }^{*}$ E-mail: martinez@cecs.cl, ratron@cecs.cl, jz@cecs.cl 
In this paper we report an exact black hole solution in four dimensions with electromagnetic and conformally coupled scalar fields. This black hole only exists for a positive cosmological constant $\Lambda$, in agreement with recent observations [1], and if a quartic selfinteraction coupling is considered. This coupling does not spoil the conformal invariance of the scalar field equation. There is a curvature singularity at the origin, and the scalar field is regular both on and outside the event horizon. The electrically charged solution exists with a fixed charge-to-mass ratio, which is given in terms of the fundamental constants of the action. This last feature implies a bound for the self-coupling constant, which is also obtained through the analysis of the solution with a nonvanishing constant scalar field.

Static scalar field configurations such as those presented here, which are regular both at the horizon as well as outisde, are unexpected in view of the no-hair conjecture (see, e.g., [2]). Furthermore, this solution can be seen to satisfy the dominant and the strong energy conditions, which corresponds to real matter under normal conditions. This is surprising in view of the recent observation that there can be no scalar hair for a positive cosmological constant and for a large class of potentials which, however, do not include the form of coupling considered here [3, 化.

The conformal coupling for the scalar field is the unique prescription that guarantees the validity of the equivalence principle in curved spacetime [5]. From a different point of view, conformally coupled scalar fields have been shown to play an important role in diverse settings of current physical interest [6, 7, 8].

For a negative cosmological constant, exact black hole solutions with a nontrivial conformally coupled scalar field, which is regular everywhere, are known only in three dimensions [9, 10].

We begin with the electrically neutral case. The action is

$$
I\left[g_{\mu \nu}, \phi\right]=\int d^{4} x \sqrt{-g}\left[\frac{R-2 \Lambda}{16 \pi G}-\frac{1}{2} g^{\mu \nu} \partial_{\mu} \phi \partial_{\nu} \phi-\frac{1}{12} R \phi^{2}-\alpha \phi^{4}\right],
$$

where $\alpha$ is a dimensionless constant $(\hbar=c=1)$.

The field equations are

$$
\begin{aligned}
& G_{\mu \nu}+\Lambda g_{\mu \nu}=8 \pi G T_{\mu \nu}, \\
& \square \phi-\frac{1}{6} R \phi-4 \alpha \phi^{3}=0,
\end{aligned}
$$

where $\square \equiv g^{\mu \nu} \nabla_{\mu} \nabla_{\nu}$, and

$$
T_{\mu \nu}=\partial_{\mu} \phi \partial_{\nu} \phi-\frac{1}{2} g_{\mu \nu} g^{\alpha \beta} \partial_{\alpha} \phi \partial_{\beta} \phi+\frac{1}{6}\left[g_{\mu \nu} \square-\nabla_{\mu} \nabla_{\nu}+G_{\mu \nu}\right] \phi^{2}-\alpha g_{\mu \nu} \phi^{4} .
$$

Since the matter part of the action is invariant under conformal transformations,

$$
g_{\mu \nu} \rightarrow \Omega^{2}(x) g_{\mu \nu}, \quad \phi \rightarrow \Omega^{-1}(x) \phi
$$

the stress tensor is traceless, and as a consequence, the scalar curvature is constant:

$$
R=4 \Lambda \text {. }
$$


The field equations are solved by the following line element:

$$
d s^{2}=-\left[-\frac{\Lambda}{3} r^{2}+\left(1-\frac{G M}{r}\right)^{2}\right] d t^{2}+\left[-\frac{\Lambda}{3} r^{2}+\left(1-\frac{G M}{r}\right)^{2}\right]^{-1} d r^{2}+r^{2} d \Omega^{2},
$$

where $0 \leq r<\infty, d \Omega^{2}$ is the metric of $S^{2}$, and the scalar field is given by

$$
\phi(r)=\sqrt{\frac{3}{4 \pi}} \frac{\sqrt{G} M}{r-G M} .
$$

This solution exists only for $\alpha=-\frac{2}{9} \pi \Lambda G$, and describes a static and spherically symmetric black hole, provided the cosmological constant $\Lambda$ is positive, and the mass satisfies $0<G M<$ $l / 4$, where the cosmological radius is $l=\sqrt{3 / \Lambda}$.

The geometry of this black hole (7) is the same as the Reissner-Nordström-de Sitter solution for the electric charge equal to the mass. The inner, event, and cosmological horizons satisfy $0<r_{-}<G M<r_{+}<\frac{l}{2}<r_{++}<l$, where

$$
\begin{aligned}
r_{-} & =\frac{l}{2}(-1+\sqrt{1+4 G M / l}), \\
r_{+} & =\frac{l}{2}(1-\sqrt{1-4 G M / l}), \\
r_{++} & =\frac{l}{2}(1+\sqrt{1-4 G M / l}) .
\end{aligned}
$$

There is a curvature singularity at the origin, and the simple pole in the scalar field is located at $r=G M$, which lies between $r_{-}$and $r_{+}$.

The massless solution has a vanishing scalar field and corresponds to de Sitter spacetime, which has a cosmological horizon at $r_{++}=l$. For the maximum allowed value of the mass, $M=l(4 G)^{-1}$, the event and cosmological horizons coalesce, $r_{+}=r_{++}=l / 2$. In case of negative mass or for $M>l(4 G)^{-1}$ the singularities become naked, thus these values of $M$ are excluded by cosmic censorship.

Note that the scalar field does not endow the black hole with a new hair because $\phi$ cannot be switched off keeping the mass fixed. In fact, $M$ is the only integration constant and for $\phi \rightarrow 0$, the geometry approaches de Sitter spacetime. Furthermore, for a given mass there are two fairly different static and spherically symmetric black hole solutions: one with $\phi=0$, which is the standard Schwarzschild-de Sitter black hole, and the new one with metric (7), and $\phi=\phi(r)$ given by Eq. (8), which only exists for positive $\Lambda$ and a quartic self-coupling with $\alpha=-\frac{2}{9} \pi \Lambda G$.

In the vanishing cosmological constant limit $\Lambda \rightarrow 0$, the geometry is described by the extremal Reissner-Nordström metric

$$
d s^{2}=-\left(1-\frac{G M}{r}\right)^{2} d t^{2}+\left(1-\frac{G M}{r}\right)^{-2} d r^{2}+r^{2} d \Omega^{2}
$$

which has coalesced inner and event horizons at $r_{+}=r_{-}=G M$. However, as the scalar field remains unchanged in this limit, it diverges at the horizon. This asymptotically flat solution 
only exists for $\alpha=0$ and was previously found independently by Bronnikov, Melnikov, and Bocharova [11], and Bekenstein [12].

- Electrically charged scalar black hole

An electrically charged black hole supporting a nontrivial scalar field is found, adding to the action the Maxwell term

$$
-\frac{1}{16 \pi} \int d^{4} x \sqrt{-g} F^{\mu \nu} F_{\mu \nu} .
$$

This static and spherically symmetric solution is described by the same metric as in Eq. (7), where the only nonvanishing component of the electromagnetic field is

$$
F_{r t}=\partial_{r} A_{t}=\frac{Q}{r^{2}}
$$

where the charge-to-mass ratio is given by

$$
\left(\frac{Q}{M}\right)^{2}=G\left(1+\frac{2 \pi \Lambda G}{9 \alpha}\right)
$$

and the scalar field reads

$$
\begin{aligned}
\phi(r) & =\sqrt{\frac{3}{4 \pi}} \frac{\sqrt{G M^{2}-Q^{2}}}{r-G M}, \\
& =\sqrt{-\frac{\Lambda}{6 \alpha}} \frac{G M}{r-G M} .
\end{aligned}
$$

Note that, Eq. (14) determines the charge-to mass ratio only in terms of the fundamental constants of the action, and implies that the bound

$$
\alpha<-\frac{2}{9} \pi \Lambda G
$$

must hold in order for the electrically charged solution to exist. This solution describes a regular black hole with a nonvanishing real scalar field provided $\Lambda>0$. Moreover, the bound (17) is saturated for the uncharged case previously discussed.

As it occurs for the uncharged case, this solution has only one independent integration constant, and thus, the scalar field does not add a new hair. In the vanishing cosmological constant limit, $\Lambda=0$, the solution with a nontrivial scalar field exists only for $\alpha=0$, and in this case the electric charge is not related to the mass. The geometry of this solution is described by the metric (12), where the electric field is given by Eq. (13), and the scalar field by Eq. (15), which diverges at the horizon. This asymptotically flat solution was previously found in Ref. 12].

It can be directly checked that the dominant and strong energy conditions hold both for the charged and uncharged solutions. Indeed, the stress energy tensor is of type I in the classification of Ref. [13]. In an orthonormal frame,

$$
\begin{aligned}
T^{a b} & =\frac{1}{8 \pi G} \operatorname{diag}\left(\frac{G^{2} M^{2}}{r^{4}},-\frac{G^{2} M^{2}}{r^{4}}, \frac{G^{2} M^{2}}{r^{4}}, \frac{G^{2} M^{2}}{r^{4}}\right) \\
& \equiv \operatorname{diag}\left(\rho, p_{1}, p_{2}, p_{3}\right) .
\end{aligned}
$$


Hence, $\rho \geq 0$, and $-\rho \leq p_{i} \leq \rho$ imply the dominant energy condition. Moreover, $\rho+p_{i} \geq 0$ $\forall i$, and $T_{a}^{a} \geq 0$ imply the strong energy condition.

- Solutions with a constant scalar field

Since the electromagnetic stress energy tensor $T_{\mu \nu}^{E}$ is traceless, and by virtue of Eqs. (3) and (6), solutions with a nonvanishing constant scalar field exist for

$$
\phi_{0}^{2}=-\frac{\Lambda}{6 \alpha},
$$

provided $\Lambda$ and $\alpha$ has opposite signs, and for any metric satisfying Einstein's equations

$$
G_{\mu \nu}+\Lambda g_{\mu \nu}=8 \pi \tilde{G} T_{\mu \nu}^{E},
$$

where $\tilde{G}$ is the effective Newton constant, given by

$$
\tilde{G}=G\left(1+\frac{2 \pi \Lambda G}{9 \alpha}\right)^{-1} .
$$

The same result is obtained in the presence of any other conformally invariant matter coupling, e.g., couplings withYang-Mills fields.

An attractive gravitational interaction requires $\tilde{G}>0$, which also gives a well defined propagator for the graviton. This implies that the bound

$$
1+\frac{2 \pi \Lambda G}{9 \alpha}>0
$$

must hold, which in the case of a negative cosmological constant means that the $\alpha>$ $-2 \pi \Lambda G / 9$, and for a positive cosmological constant, the bound (17), imposed by the chargeto-mass ratio, is recovered from a different point of view.

\section{Acknowledgments}

Useful discussions with Andrés Gomberoff, Mokhtar Hassaïne and Claudio Teitelboim are gratefully acknowledged. This work is partially funded by FONDECYT grants 1020629, 1010446, 1010449, 1010450, 7010446, and 7010450, and from the generous support to CECS by Empresas CMPC. CECS is a Millennium Science Institute.

\section{References}

[1] Supernova Cosmology Project Collaboration, S. Perlmutter et al., Nature (London) 391, 51 (1998); Astrophys. J. 517, 565 (1999).

[2] M. Heusler, Black Hole Uniqueness Theorems (Cambridge University Press, Cambridge, England, 1996).

[3] R.-G. Cai and J.-Y. Ji, Phys. Rev. D 58, 024002 (1999).

[4] E. Winstanley, On The existence of conformally coupled scalar hair for black holes in (anti-) de Sitter space, e-print Archive: hep-th/0205092. 
[5] V. Faraoni and S. Sonego, Class. Quantum Grav. 10, 1185 (1993).

[6] C.G. Callan, S.R. Coleman, and R. Jackiw, Ann. Phys. (N.Y.) 59, 42 (1970).

[7] P.J.E. Peebles and A. Vilenkin, Phys. Rev. D 59, 063505 (1999).

[8] N. Seiberg and E. Witten, J. High Energy Phys. 04, 017 (1999).

[9] C. Martínez and J. Zanelli, Phys. Rev. D 54, 3830 (1996).

[10] M. Henneaux, C. Martínez, R. Troncoso, and J. Zanelli, Phys. Rev. D 65, 104007 (2002).

[11] N. Bocharova, K. Bronnikov, and V. Melnikov, Vestn. Mosk. Univ. Fiz. Astron. 6, 706 (1970).

[12] J.D. Bekenstein, Ann. Phys. (N.Y.) 82, 535 (1974); 91, 75 (1975).

[13] S. W. Hawking and G. F. R. Ellis, The Large-Scale Structure of Space-Time (Cambridge University Press, Cambridge, England, 1973), Sec. 4.3. 